\title{
Tendances actuelles dans la mesure de l'exposition professionnelle aux fibres
}

\author{
P. O. Droz ${ }^{1}$, G. Litzistorf ${ }^{2}$, M. Guillemin ${ }^{l}$ \\ 1 Institut universitaire de médecine du travail et d'hygiène industrielle, Rte de la Clochatte, CH-1052 Le Mont \\ 2 Institut interdépartemental de microscopie électronique de T'EPF-L, CH-1015 Lausanne
}

Il a été montré depuis de nombreuses années que les fibres d'amiante inhalées peuvent provoquer des effets toxiques sérieux (asbestose, cancer pulmonaire, mésothel iome). De plus, l'importance de ces effets est fonction de la nature d'amiante en cause, comme en témoignent les "Threshold Limit Values" (TLV) présentées dans le tableau I.

Tableau I : Threshold Limit Values (TLV 1984 ACGIH) pour quelques types d'amiantes

\begin{tabular}{lclc}
\hline Amiante & TLV $[\mathrm{f} / \mathrm{ml}]^{*}$ & Amiante & TLV $[\mathrm{f} / \mathrm{ml}]^{\star}$ \\
\hline chrysotile & 2 & antophyllite & 2 \\
crocidolite & 0.2 & actinolite & 2 \\
amosite & 0.5 & tremolite & 2 \\
\hline
\end{tabular}

* fibres long. $>5 \mu \mathrm{m}$, diam. $<3 \mu \mathrm{m}$

D'autre part, Pott a montré que le pouvoir cancérigène des fibres dépend aussi de leur forme géométrique (longueur, diamètre), les petites étant moins actives par exemple.

L'évaluation du risque d'exposition aux fibres d'amiante apparait donc comme une démarche très complexe. Idéalement, il s'agirait de disposer de techniques permettant à la fois de caractériser la nature et les dimensions des fibres en question.

Traditionnellement, l'exposition professionnelle aux fibres d'amiante est mesurée par la méthode optique à contraste de phase. Les fibres, prélevées sur un filtre en esters de cellulase, sont comptées après montage, à l'aide d'un microscope à contraste de phase. Sont comptés comme fibres tous les objets ayant un rapport longueur/diamètre de $3 / 1$ (voire $5 / 1$ actue 7 lement), ayant une longueur supérieure à $5 \mu \mathrm{m}$ et un diamètre < $3 \mathrm{um}$. Le comptage des fibres fines est limité par le pouvoir de résolution du microscope qui est de $0.2 \mu \mathrm{m}$. En général un minimum de 100 fibres sont comptées pour des raisons statistiques.

Cette méthode, qui est la seule pouvant étre utilisée pour l'application des valeurs TLV du tableau I, présente plusieurs désavantages :

- elle n'est pas spécifique, puisqu'elle ne compte que des objets ayant une forme de fibre;

- elle n'est qu'un indicateur grossier de la concentration totale de fibres dans l'air (généralement seuls quelques pourcents de toutes les fibres sont observables au microscope optique) ;
- sa limite de détection reste élevée (dans les meilleurs cas $0.01 \mathrm{f} / \mathrm{ml}$ ) par rapport aux valeurs trouvées actuellement dans les baatiments floqués, par exemple;

- l'analyse est relativement lente, elle ne donne pas d'indication directe, qui pourrait être très utile dans certains cas;

- on n'obtient par cette technique qu'ure valeur moyenne sur la durée de prélèvement.

Pour pallier ces différents inconvénients, deux voies de développement sont apparues depuis plusieurs années déjà: d'une part l'emploi de diverses techniques de microscopie électronique (SEM, TEM liés aux analyses SAED ou EDXA) pour améliorer la spécificité, la sensibilité et la résolution; d'autre part le développement d'une instrumentation à lecture directe pour faciliter l'acquisition de réponses immédiates (Fibrous Aerosol Monitor - FAM - GCA Corporation).

Les possibilités techniques et les coûts de ces différentes méthodes sont résumés dans les tableaux II et III. Par comparaison de ces deux tableaux, on constate a isément que 1 'augmentation des exigences techniques d'une méthode provoque un accroissement parallèle des coûts. Il s'agit donc d'établir un compromis coūt/résultats qui va dépendre du type d'environnement cons $i-$ déré, conme cela est présenté de façon résumée dans le tableau IV.

Tableau II : caractéristiques techniques des differentes méthodes

\begin{tabular}{|c|c|c|c|c|c|}
\hline Méthode & $\begin{array}{l}\text { Type } \\
\text { forme }\end{array}$ & $\begin{array}{l}\text { informat } \\
\text { minéra- } \\
\text { logique }\end{array}$ & $\begin{array}{l}\text { ion } \\
\text { chimi- } \\
\text { que }\end{array}$ & $\begin{array}{l}\text { Limite de } \\
\text { détection } \\
{[f / 1]^{\star}}\end{array}$ & $\begin{array}{l}\text { e Réso- } \\
\text { n lution } \\
\text { [nm] }]^{\star *}\end{array}$ \\
\hline $\begin{array}{l}\text { Optique } \\
\text { SEM/EDXA } \\
\text { TEM/SAED, EDXA } \\
\text { FAM }\end{array}$ & $\begin{array}{l}+ \\
+ \\
+ \\
+\end{array}$ & $\begin{array}{l}- \\
- \\
+\end{array}$ & $\begin{array}{l}- \\
+ \\
+ \\
-\end{array}$ & $\begin{array}{l}10 \\
0.1 \\
0.1 \\
1\end{array}$ & $\begin{array}{r}200 \\
200 \\
50 \\
-\quad 200\end{array}$ \\
\hline \multicolumn{6}{|c|}{$\begin{array}{l}\text { * fibres }>5 \mu \mathrm{m} \quad * \star \text { atteignable en routine } \\
\text { Tableau III : caractéristiques économiques des } \\
\text { différentes méthodes }\end{array}$} \\
\hline $\begin{array}{l}\text { Durée } \\
\text { lyse }\end{array}$ & $\begin{array}{l}d^{\prime} \text { ana } \\
{[\mathrm{h}]}\end{array}$ & $\begin{array}{l}\text { Coût } \\
\text { lyse }\end{array}$ & $\begin{array}{l}\text { ar ana- } \\
{[\mathrm{fr} .]}\end{array}$ & $\begin{array}{r}\text { Investis } \\
{[\mathrm{fr}}\end{array}$ & $\begin{array}{l}\text { ssement } \\
.]\end{array}$ \\
\hline $\begin{array}{l}\text { Optique } \\
\text { SEM/EDXA } \\
\text { TEM/SAED,EDXA } \\
\text { FAM }\end{array}$ & $\begin{array}{l}1 \\
4 \\
6 \\
0\end{array}$ & $\begin{array}{r}1 ' \\
3 \prime \\
\text { fait }\end{array}$ & $\begin{array}{l}100 \\
000 \\
00 \\
0\end{array}$ & $\begin{array}{r}12^{\prime} \\
200^{\prime} \\
600^{\prime} \\
30^{\prime}\end{array}$ & $\begin{array}{l}000 \\
000 \\
000 \\
000\end{array}$ \\
\hline
\end{tabular}

Tableau IV: caractéristiques des différents environnements et méthodes proposées

\begin{tabular}{|c|c|c|c|c|c|c|c|}
\hline Environnement & $\begin{array}{c}C \text { a } r \text { a } c t \\
\text { Nature fibres }\end{array}$ & $\begin{array}{l}r \text { é } \\
\text { concentration }\end{array}$ & $\begin{array}{l}\text { e s } \\
\text { Norme }\end{array}$ & $\begin{array}{r}M \text { é } \\
\text { Optique }\end{array}$ & $\begin{array}{l}\text { ho } \\
\text { SEM }\end{array}$ & $\begin{array}{l}\text { es } \\
\text { TEM }\end{array}$ & FAM \\
\hline $\begin{array}{l}\text { Professionnel } \\
\text { Paraprofessionnel } \\
\text { Extérieur }\end{array}$ & $\begin{array}{l}\text { connue } \\
\text { mal connue } \\
\text { inconnue }\end{array}$ & $\begin{array}{l}\text { forte } \\
\text { faible } \\
\text { très faible }\end{array}$ & $\begin{array}{c}\text { MAC, TLV } \\
-\overline{V I}\end{array}$ & $\begin{array}{l}+ \\
-\end{array}$ & $\begin{array}{l}(+) \\
+ \\
+\end{array}$ & $\begin{array}{l}- \\
(+) \\
+\end{array}$ & $\begin{array}{l}+ \\
+ \\
-\end{array}$ \\
\hline
\end{tabular}

